\title{
Seeing emotions in the eyes - inverse priming effects induced by eyes expressing mental states
}

\section{Caroline Wagenbreth, Julia Rieger, Hans-Jochen Heinze and Tino Zaehle*}

Department of Neurology, Otto-von-Guericke University Magdeburg, Magdeburg, Germany

\section{Edited by:}

Marina A. Pavlova, Eberhard Karls

University of Tübingen, Germany

\section{Reviewed by:}

Olivier Piguet, Neuroscience Research Australia, Australia

Mariska Esther Kret, University of Amsterdam, Netherlands

\section{*Correspondence:}

Tino Zaehle, Department of Neurology, Otto-von-Guericke University Magdeburg, Leipziger Straße 44, 39120 Magdeburg, Germany

e-mail: tino.zaehle@ovgu.de
Objective: Automatic emotional processing of faces and facial expressions gain more and more of relevance in terms of social communication. Among a variety of different primes, targets and tasks, whole face images and facial expressions have been used to affectively prime emotional responses. This study investigates whether emotional information provided solely in eye regions that display mental states can also trigger affective priming.

Methods: Sixteen subjects answered a lexical decision task (LDT) coupled with an affective priming paradigm. Emotion-associated eye regions were extracted from photographs of faces and acted as primes, whereas targets were either words or pseudo-words. Participants had to decide whether the targets were real German words or generated pseudo-words. Primes and targets belonged to the emotional categories "fear," "disgust," "happiness," and "neutral."

Results: A general valence effect for positive words was observed: responses in the LDT were faster for target words of the emotional category happiness when compared to other categories. Importantly, pictures of emotional eye regions preceding the target words affected their subsequent classification. While we show a classical priming effect for neutral target words - with shorter RT for congruent compared to incongruent prime-target pairs-, we observed an inverse priming effect for fearful and happy target words - with shorter RT for incongruent compared to congruent prime-target pairs. These inverse priming effects were driven exclusively by specific prime-target pairs.

Conclusion: Reduced facial emotional information is sufficient to induce automatic implicit emotional processing. The emotional-associated eye regions were processed with respect to their emotional valence and affected the performance on the LDT.

Keywords: affective priming, lexical decision task, read the mind in the eyes, valence, face

\section{INTRODUCTION}

Due to the important role of facial features in social communication, effects arising from automatic affective processing of faces and facial expressions gain more and more of interest (Li et al., 2008; Suslow et al., 2010; Andrews et al., 2011; Rohr et al., 2012; Sassi et al., 2014). Faces are complex, concrete and socially significant stimuli that are linked to emotional reactions (Murphy and Zajonc, 1993). In addition, a human face holds a natural salience, i.e., it is an interesting stimulus that attracts attention more than other visual stimuli (Krebs et al., 2011). A faster detection of fearful or angry when compared to neutral faces (Ishai et al., 2004) as well as an attentional bias to threatening facial expressions could be observed (Susa et al., 2012). Moreover, a facilitation of a visual search task to identify fear-related pictures among fear-irrelevant ones was shown (Öhman et al., 2001) as well as a slower attention disengagement from angry faces compared to neutral or happy ones (Fox et al., 2002). In agreement with the evolutionary point of view this suggests a faster processing of threatening or possibly life-endangering stimuli. Thus, decoding and interpreting emotions displayed in facial expressions, especially those with negative valence, play a fundamental role in human interactions.
Identifying emotional states of other persons also constitutes the basis of the social construct of theory of mind (ToM). ToM refers to the process of emotion recognition and allows individuals to imagine and attribute the mental states of others (Pinkham et al., 2003). The "Reading the mind in the eyes task" (RMET) which was invented by Baron-Cohen et al. (1997) is an advanced test of ToM and consists of photographs showing the facial region around the eyes. Four different emotional states are offered and the participant is asked to decide which of these emotional states describes best what the person on the photograph is thinking or feeling. Thus, the RMET is a social cognition measure to evaluate the participant's ability to recognize and identify mental states and their intensity. It refers to explicit emotional processing which requires conscious action under deliberate control. In contrast, implicit processing of emotions is an automatic process that does not allow for the activation of expectancies or response strategies and is thus independent of cognitive resources. Implicit - or automatic-activation of emotional evaluations is often measured in affective priming paradigms (Fazio, 2001; Klauer and Musch, 2003). Here, the automatically triggered connection between two stimuli (prime and target) which are determined through 
their emotional valence is essential. Faster responses and fewer errors were observed when two consecutively presented stimuli are identical (congruent) with respect to their emotional valence (e.g., positive-positive) than when they differ from each other (Andrews et al., 2011). This congruence effect has been shown to be a robust and replicable phenomenon in a variety of studies using different primes, targets and specific instructions and tasks (Hermans et al., 1994, 1998, 2003; De Houwer et al., 1998; Giner-Sorolla et al., 1999; Banse, 2001). The effect of congruence between prime and target allows for the systematic evaluation of the automatic emotional processing that is triggered by the prime.

In affective priming paradigms, the most commonly used task for the participants is to evaluate a target concerning its valence, as applied in a lexical decision task (LDT). The LDT is a common instrument to measure implicit emotional processing since it focuses on a non-emotional decision which is not distorted by emotional features of the stimuli. In a LDT, participants have to judge the lexical status of a presented letter string on whether it is a correct real word or a pseudo- or nonword. To ensure an "affective" influence on the LDT, words with different emotional valences are used in this task. Hence, valence is an important manipulating variable on the performance in LDTs. Different studies showed shorter reaction times (RT) and fewer errors of participants for emotional when compared to neutral words (Challis and Krane, 1988; Williamson et al., 1991; Windmann et al., 2002; Nakic et al., 2006; Schacht and Sommer, 2009). Positive valence is known to facilitate lexical processing in the LDT (Kuchinke et al., 2005, 2007; Schacht and Sommer, 2009; Scott etal., 2009) while negative valence itself seems to slow RT (Briesemeister et al., 2011a). This effect on RT for negatively valenced words is observed only at high levels of emotional arousal (Nakic et al., 2006; Larsen et al., 2008; Hofmann et al., 2009), which suggests a simplified and preferred processing of for instance fearful words that are expected to have a high emotional arousal.

In recent studies that applied affective priming paradigms, whole face images and facial expressions have been used as primes since facial features are supposed to be recognized ubiquitously as indicators of different affective conditions (Li et al., 2008; Suslow et al., 2010; Andrews et al., 2011; Rohr et al., 2012; Sassi et al., 2014). However, it is not clear whether these findings are also true for reduced emotional information that is featured not in the whole face, but solely in the eye regions of a face expressing mental states. Therefore, in the present study we systematically investigated the ability of emotional eyes expressions to induce implicit emotional processing by using an affective priming paradigm. We assume that even reduced emotional information in human eyes is sufficient to influence automatic emotional responses. Here, eyes expressing mental states acted as primes to influence the subsequent answer on a target word in a LDT.

\section{MATERIALS AND METHODS SUBJECTS}

Sixteen healthy participants (female $=8$ ) in the age of 2228 years (mean age $=24.12 \pm 1.54$ years) volunteered in this study. All participants were German native speakers, right-handed, reported normal, or corrected-to-normal vision and were free of any neurological or psychiatric impairment. The experiment was approved by the local ethics committee (University of Magdeburg, Germany).

\section{MATERIAL}

For the LDT a set of 192 written stimuli (96 German words and 96 pseudo-words) were used. Neutral words were extracted from the "Berlin Affective Word List Reloaded" (BAWL-R; Võ et al., 2009) when they showed a valence rating of 0 . Emotional words were taken from the "discrete emotion norms for nouns: Berlin affective word list” (DENN-BAWL; Briesemeister et al., 2011b). Here, only those words were chosen that achieved an emotional intensity score of at least 3 on a Likert-scale according to Briesemeister et al. (2011b). All 96 words were grouped in four emotional categories: 24 fear-related [e.g., "PANIK"; ("PANIC")], 24 disgustrelated [e.g., "PARASIT"; ("PARASITE”)], 24 happiness-related [e.g., "LIEBE"; ("LOVE")] and 24 neutral words [e.g., "WOCHE"; ("WEEK")]. Pseudo-words were also generated out of the words listed in the BAWL-R. Here, neutral words with a length of four to eight letters were manipulated by interchanging single vowels or consonants within this word. By doing so, new pronounceable but meaningless letter strings were created (e.g., "POLITIK" "PILITOK”).

Facial region around the eyes extracted from the stimulus set "60 Faces Test" developed by Ekman and Friesen (1976) were used as emotional primes. This set consists of the six basic human emotional facial expressions (happiness, sadness, disgust, fear, surprise, and anger as well as a neutral condition). For the present study, we chose four different persons out of this set (two female, two male) and selected their relevant facial expressions on a picture (happiness, fear, disgust, neutral). The whole-face images were then adjusted and cut so that only the eye regions were visible. After this editing, the resulting 16 prime pictures were presented in a size of $8.6 \mathrm{~cm} \times 3 \mathrm{~cm}$ (3.4 visual angle).

Primes (eye region pictures) and target stimuli (words or pseudo-words) were combined in a pseudo-randomized manner: half of the prime-target pairs of the real German words were constructed to be congruent. The sequence of combination is displayed in Table 1.

\section{TASK}

The experiment was generated and carried out with presentation (Neurobehavioral Systems, Inc.). Participants were seated in front of a computer and were requested to look at a fixation cross in the middle of the screen. The task consisted of 192 trials and included a break after the half of the trials (96); the participants could decide how long this break would last. Depending on the understanding of instruction and the length of the break, the duration of the whole task was about $15 \mathrm{~min}$.

Each trial started with a fixation cross that was shown for $500 \mathrm{~ms}$ before an emotional prime was displayed for $150 \mathrm{~ms}$. After a short pause (inter-stimulus interval of $50 \mathrm{~ms}$ ) the target stimulus was presented for a maximum of $3000 \mathrm{~ms}$ or until a response was made. During this time interval, the participants had to decide whether this target was a real word or a pseudo-word by pressing the corresponding button on the mouse. Afterward, the next trial 
Table 1 | Division of the prime-target word-pairings in the lexical decision task (LDT) according to congruence.

\begin{tabular}{lllll}
\hline Congruence & \multicolumn{2}{c}{ Combinations } & $\begin{array}{l}\text { Number per } \\
\text { pair }\end{array}$ & $\begin{array}{l}\text { Total } \\
\text { number }\end{array}$ \\
\cline { 2 - 3 } & \multicolumn{2}{c}{ Prime } & Target word & \\
\hline \multirow{4}{*}{ Incongruent } & Fear & Fear & 12 & 48 \\
& Disgust & Disgust & 12 & \\
& Happiness & Happiness & 12 & \\
& Neutral & Neutral & 12 & \\
& Fear & Disgust & 4 & \\
& Fear & Happiness & 4 & \\
& Fear & Neutral & 4 & \\
& Disgust & Fear & 4 & \\
& Disgust & Happiness & 4 & \\
& Disgust & Neutral & 4 & \\
& Happiness & Fear & 4 & \\
& Happiness & Disgust & 4 & \\
& Happiness & Neutral & 4 & \\
& Neutral & Fear & 4 & \\
& Neutral & Disgust & 4 & \\
& Neutral & Happiness & 4 & \\
& & & & \\
\hline
\end{tabular}

started (see Figure 1). The presentation order of items (primes and targets) as well as the assignment of mouse buttons to respond was counterbalanced across subjects.

\section{ANALYSIS}

Mean RT and error rates for each participant were assessed and entered into a $4 \times 2$ repeated-measures ANOVA with the factors valence of the target word (fear, disgust, happiness, neutral) and emotional congruence of the prime-word pairing (congruent, incongruent). Subsequently individual prime-target pairings were evaluated separately. Prior to analysis RT normal distribution was confirmed using the Kolmogorov-Smirnov Test of Normality $(p=0.89)$. All results are displayed in mean $\pm \mathrm{SD}(\mathrm{M} \pm \mathrm{SD})$.

\section{RESULTS}

A repeated-measures ANOVA on RT showed a significant main effect of the factor valence $[F(3,45)=14.84 ; p<0.001]$, no main effect of the factor congruence $[F(1,15)=0.27 ; p=0.61]$, and a significant valence $\times$ congruence interaction $[F(3,45)=9.04$; $p<0.001]$. The main effect of valence was driven by significantly shorter RT for happiness-related target words compared to all other emotional categories (all $p<0.001$; see Figure 2A).

The valence $\mathrm{x}$ congruence interaction was further investigated by comparing incongruent and congruent trials separately for each emotional target word category. Pairwise $t$-tests revealed shorter RT for incongruent prime-target pairs compared to congruent pairs for the word categories fear $[T(15)=2.35 ; p<0.05]$ and happiness $[T(15)=2.67 ; p<0.05]$, and shorter RT for congruent pairs compared to incongruent pairs $[T(15)=-2.66$; $p<0.05$ ] for neutral targets (see Figure 2B). No effects were visible for the emotion category disgust $[T(15)=-1.08 ; p=0.3]$. Thus, whereas there was a classical priming effect for neutral target words with a processing advantage for congruent pairs, we revealed an inverse priming effect for fearful and happy target words with an advantage for the processing of incongruent primed targets.

A repeated-measures ANOVA on error rate showed a nonsignificant statistical trend for the factor valence $[F(3,45)=2.44$; $p=0.07]$, no effect of the factor congruence $[F(1,15)=1.36$; $p=0.26]$, and no significant valence $\mathrm{x}$ congruence interaction $[F(3,45)=0.67 ; p=0.57]$. The trend for the main effect of valence was driven by significantly more errors for disgust-related words compared to happiness-related words ( $p=0.05$; see Figure 3A).

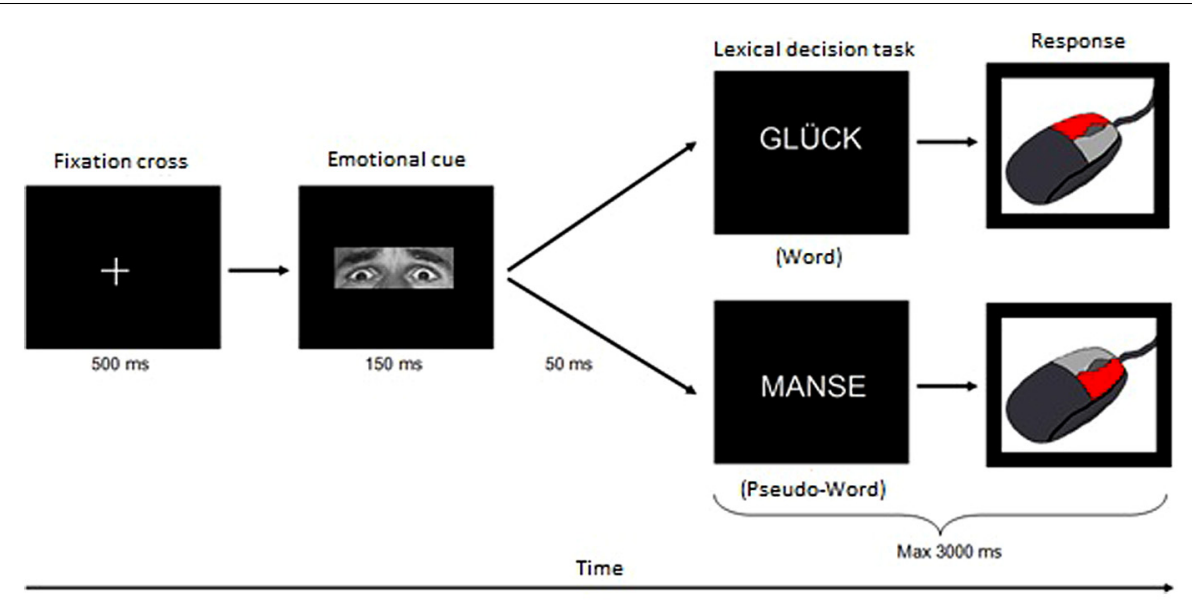

FIGURE 1 | Experimental design of the implicit emotional processing with LDT. Each trial started with a fixation cross that was presented for $50 \mathrm{~ms}$. Subsequently an emotional prime (the adjusted eye region of the Ekman-faces) was displayed for $150 \mathrm{~ms}$ followed by a break of $50 \mathrm{~ms}$. Finally, the target stimulus was presented. The participants were supposed to decide as fast as possible whether this target was a real German word or a pseudo-word and to press the corresponding button on the mouse within $3000 \mathrm{~ms}$. After this time interval the next trial started. 


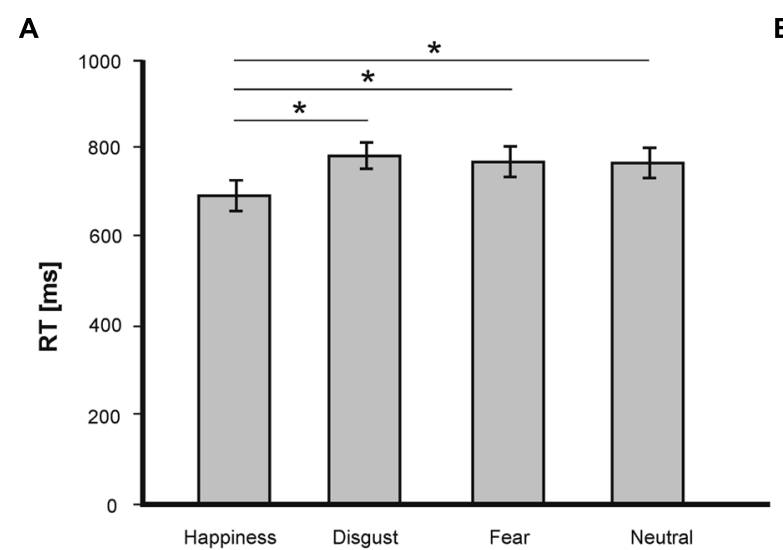

FIGURE 2 | (A) Mean RT (in ms) in the LDT for target words of the emotional categories fear, disgust, happiness and neutral. Statistically significant differences are labeled with $\left({ }^{*}\right)$. Error bars display SEs. (B) Mean RT (in ms) in

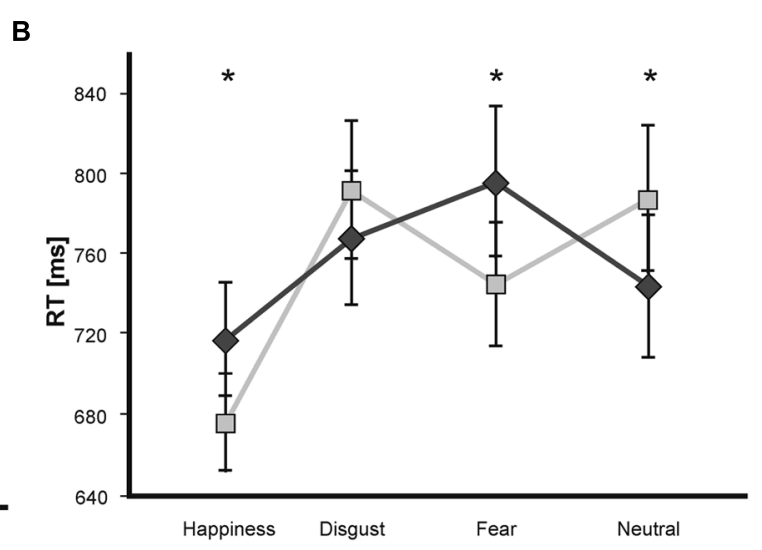

the LDT as a function of valence and congruence. Statistically significant differences are labeled with $\left(^{*}\right)$. Dark gray line depicts congruent trials, light gray line depicts incongruent trials.

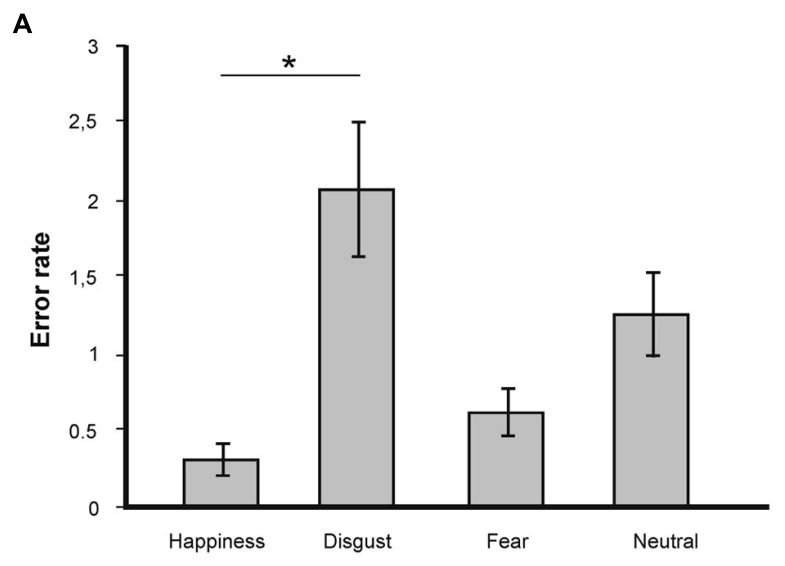

FIGURE 3 | (A) Mean number of errors in the LDT for target words of the emotional categories fear, disgust, happiness, and neutral. Statistically significant differences are labeled with $\left({ }^{*}\right)$. Error bars display SEs. (B) Mean
B

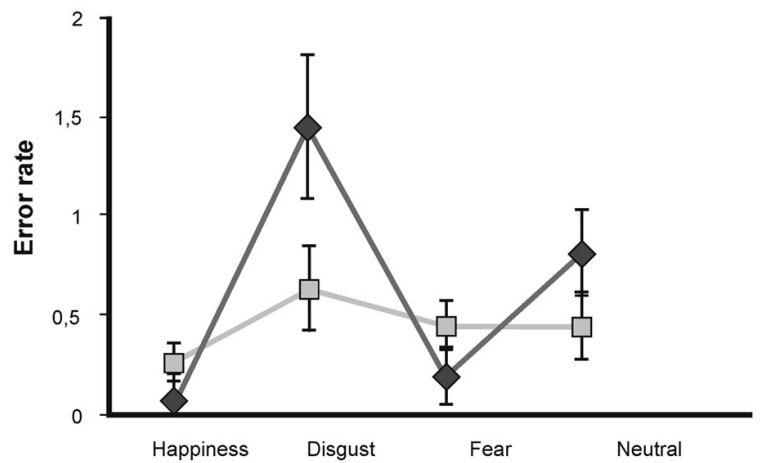

number of errors in the LDT as a function of valence and congruence. Statistically significant differences are labeled with $\left({ }^{*}\right)$. Dark gray line depicts congruent trials, light gray line depicts incongruent trials.
Further analysis showed no differences in error rate for incongruent versus congruent conditions for the emotional word categories (happiness: [ $T(15)=0.44 ; p=0.67]$; fear: $[T(15)=0.0$; $p=1.0]$; disgust: $[T(15)=0.22 ; p=0.83]$; neutral $[T(15)=1.86$; $p=0.08]$; see Figure 3B).

To further assess the observed priming effects, we analyzed responses to individual primes-targets pairs separately (see Figure 4).

For happy target words, analysis revealed that compared to the congruent (happy-happy) pairs only the incongruent fear-happy pairs was answered significantly faster $[T(15)=-5.73 ; p<0.001]$. For fearful target words, analysis showed that the neutral-fear pairs were answered significantly faster that the congruent (fearfear) pairs $[T(15)=-4.62 ; p<0.001$; see Figure 4]. No further congruent-incongruent comparison revealed statistical significance.

In sum, the valence of the target words had a significant effect on the RT in the LDT. Happiness-related target words were answered faster than words of all other emotional categories. Importantly, the emotional content of the facial expressions (primes) modulated the LDT responses. While we could show a classical priming effect for neutral target words - with shorter RT for congruent compared to incongruent prime-target pairs-, we observed an inverse priming effect for fearful and happy target words - with shorter RT for incongruent compared to congruent prime-target pairs. Furthermore, this inverse priming effect for happy targets was driven exclusively by the incongruent combination with fearful primes, whereas for fearful targets it was driven by neutral primes only.

\section{DISCUSSION}

\section{EFFECT OF VALENCE}

Independent from the prime-target pairing, we found a general effect of valence in the LDT with shorter RT for emotional target words when compared to neutral words. Thus, an advantage in the processing of positive words (category happiness) in form 


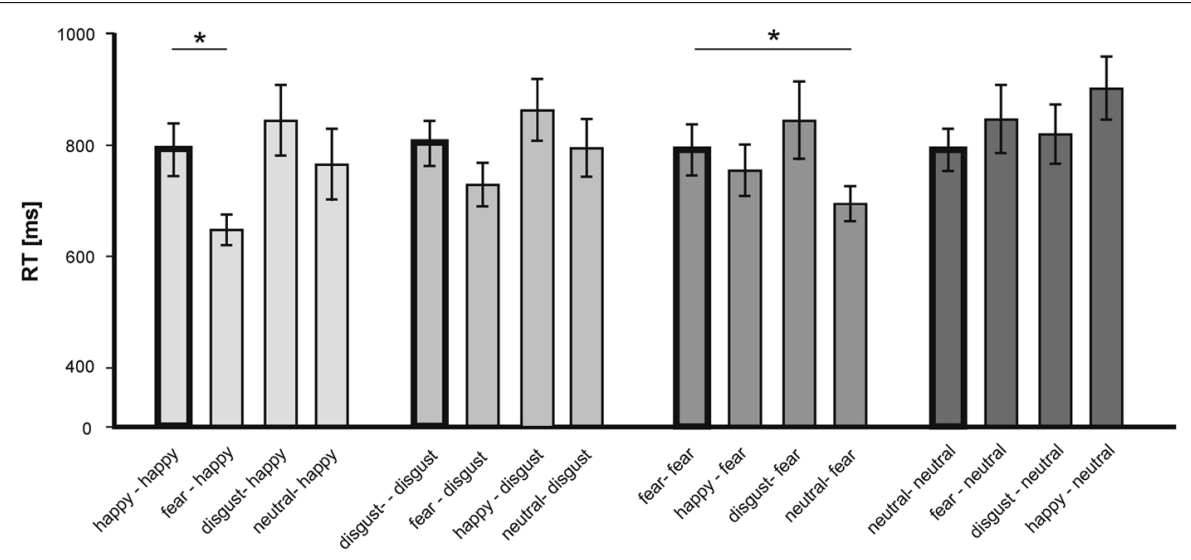

FIGURE 4 | Mean RT (in ms) in the LDT for single emotional prime-target pairings according to the valence of the target. Statistically significant differences are labeled with $\left({ }^{*}\right)$. Error bars display SEs.

of shorter RT when compared to fearful, disgust-related or neutral words was observed. However, the other categories (fear, disgust) did not differ significantly from neutral words. These findings are in good agreement with previous studies that postulate an advantageous processing of positive words (Kuchinke et al., 2005; Hofmann et al., 2009; Briesemeister et al., 2011a; Kissler and Koessler, 2011). Slowing in RT for the processing of negative words has been described recently (Wentura et al., 2000; Castner et al., 2007). In our study this increase of RT for negative words was significant when compared to positive, but not when compared to neutral words. These findings can be attributed to the "automatic vigilance" hypothesis implying that people tend to focus their attention preferentially on negative stimuli and can also rather difficult dissolve it from them (Wentura et al., 2000; Öhman and Mineka, 2001). This is in line with findings by Estes and Verges (2008) who postulated a slower disentanglement of attention from negative stimuli and with an approach by Larsen et al. (2008) who suggest a RT slowing for low arousing negative words.

\section{EFFECT OF CONGRUENCE}

We observed an interaction effect between valence and congruence which demonstrates that the influence of emotional congruency depends on the valence of the target word. A general effect of congruence between prime and target, displayed in shorter RT and fewer errors in congruent when compared to incongruent trials, is often reported in affective priming paradigms (Banse, 2001; De Houwer et al., 2009). Interestingly, the present results confirm this effect only in part. The advantage of affectively congruent primed trials was found for neutral words only and selectively for RT and not for the error rate. Contrary to the expectations, we found an inverse priming effect in terms of an advantageous processing of incongruent trials: for the emotional categories fear and happiness RT were shorter for incongruent than for congruent trials.

Different explanations have been suggested for the inverse priming effect (Glaser and Banaji, 1999; Banse, 2001; Verleger et al., 2004; Mattler, 2007). Glaser and Banaji (1999) underline the modulating character of the extremeness of primes that would provoke an automatic and unconscious correction of information processing. Following their argumentation, primes of extreme valence have the potential to distort the answer on a target. Thus, the emotional processing of "extremely valenced" primes (e.g., fearful or happy) would be increased and a proper lexical decision would require more time. In contrast, neutral primes have lower salience and no correction of distorting influences is necessary, which is reflected in shorter RT. Critically, this approach highlights the importance of the emotional information content of the primes. However, the role of the valence of targets for emotional processing is not affected and thus this explanation does not sufficiently account for the present findings.

Another approach is given by Maier et al. (2003). They suggested the existence of activation dependent inhibitory processes in memory. According to the authors, a strengthening of some examples of a category (i.e., either positive or negative) is connected with an automatic inhibition of related, but interfering examples of the same category. For instance, as soon as the representation of a positive stimulus as "positive" is enhanced, automatic activation processes start inhibiting stimuli of the same category ("positive"). Effects of congruency would lose ground in favor of automatic inhibitory processes and the accessibility for an incongruent target word would thus increase. This could explain RT advantages of incongruent pairs of the category happiness and fear, but not why RT are faster for incongruent neutral word trials.

Based on different explanatory attempts one might suggest a concluding approach that integrates different theories. The basic assumption here is that the valence of the primes and targets is processed unconsciously and that correction and inhibition processes are involved in affective priming. In this hypothesis, the presentation of an emotional prime activates the appropriate concept node in the semantic network (De Houwer and Hermans, 1994). According to Maier et al. (2003) the corresponding representation in the greater valence system (e.g., the concept "negative") is enhanced through automatic activation dispersion. If a congruent target stimulus follows, a stronger activation of the belonging 
memory network is run due to the additive effect of the valence information of both prime and target (Dagenbach and Carr, 1994) which results in longer RT for congruent trials. For incongruent trials, both valence representations are activated in memory, but each system apart has only a low activation level. Thus, the automatic correction system for the distortive influence of emotional information (Glaser and Banaji, 1999; Klapp, 2005) can be inhibited in favor of an adequate response. Since the activation levels of both representations are low, the activation of both valence systems is faster which results in shorter RT for incongruent trials. Thus, the strength of the activation levels determines the strength of the activation of the automatic correction system which in turn is expressed in RT. For neutral words that do not carry emotional information, no activation of greater valence systems is necessary as well as no corrective efforts. Thus, RT for neutral congruent prime-target pairs (which have no influence of relevant emotional information) are shorter than RT of neutral incongruent pairs.

Beyond the general inverse priming effects for happiness and fear, the present data show that the processing advantage for incongruently primed happy targets was mainly driven by the fearful primes. The combination of fear (prime) and happiness (target) was answered faster than all other conditions. This implies a special role of fear as characteristic for a prime. In general, fear expressions have been shown to reveal stronger subliminal priming effects than other negative emotional expressions as for instance disgust (Murphy and Zajonc, 1993; Lee et al., 2011). Fear is thus suggested to activate subliminal processing as an alarm for possible threat. In our study, the pairing fear-happy was answered fastest. It might be suggested that the fear primes acted as threat signals and increased attentional vigilance. Thus, fearful faces are processed more quickly during encoding and are more available for subsequent processing (Suslow et al., 2006). However, since happy stimuli convey quite clear and undoubted safety signals, the emersion of a happy word might resolve the alertness and elicit faster RT.

But advantage in fear processing does not only apply to primes but also to targets. Interestingly, negative stimuli (such as fear-associated) are reported to elicit longer RT than positive ones (Castner et al., 2007). And in general, fearful expressions are detected more quickly than neutral or happy expressions (Yang et al., 2007; Wang et al., 2014). In our study, we detected a fast processing of fear target words that were preceded by neutral primes, which determined the general inverse priming effect for fearful targets. The neutral facial expression corresponds to the normal, everyday facial expression that one is most accustomed to when looking in faces of other persons. No emotional processing is necessary when assessing neutral expressions. We assume that the discrepancy between this neutral expression and a sudden appearance of a threatening fear target word leads to fast responses, especially when compared to other emotional facial expressions that always imply emotional anticipation.

Affective information is not distributed uniformly in the face. There is agreement that the recognition of fearful and angry faces depends more on information in the eye region, while disgust is conveyed mainly by the mouth (Calder et al., 2000; Leppänen and Hietanen, 2007; Calvo and Marrero, 2009). In contrast to the emotions fear or happiness, the emotional category disgust captures an exceptional position. When analyzing the influence of the targets' valences on performance, most errors were detected for disgust-related target words. At the same time, no differences between congruent and incongruent conditions for disgust target words could be detected. Thus, for target words of this category no priming effect was present. Recent findings support the special role of disgust in comparison to other emotions (Sprengelmeyer et al., 1996; Phillips et al., 1997; Wang et al., 2003; Montagne et al., 2006; Hayes et al., 2007; Lee et al., 2011; Baggio et al., 2012; Borg et al., 2012; Buxton et al., 2013) indicating disgust as a negative but nonetheless non-threatening emotion in social contexts. Moreover, disgust is a highly-evaluative emotion that is predominantly affected through the culture a society lives with. Thus, there are considerable differences in what people sense or perceive as "disgusting." The choice of disgust-associated stimuli therefore constitutes a challenging task for affective priming paradigms to prevent ambiguity and future studies should consider this point carefully.

\section{LIMITATIONS}

Despite the strength of this study to demonstrate that eye regions of a face displaying mental states elicit affective priming, some confining points have to be taken into account. We present data from a rather small and very homogeneous sample concerning age and educational background. Future studies should consider more variability to report different characteristics of affective priming during implicit emotional processing. Moreover, the words used in this investigation were not selected with respect to an equally distributed word frequency. We cannot exclude possible influences of this variable on the results. However, recent studies could demonstrate RT advantage of positive words independent from the word frequency (Kuchinke et al., 2007) which underlines our findings.

\section{CONCLUSION}

To our knowledge, this is the first study demonstrating that even incomplete facial information induce implicit emotional responses and - in consequence - influence subsequent explicit decisions. These emotion-associated eye regions were processed with regard to their emotional valence and affected the performance on the following LDT. The primes used here clearly demonstrated their effectiveness in affective priming.

\section{REFERENCES}

Andrews, V., Lipp, O. V., Mallan, K. M., and König, S. (2011). No evidence for subliminal affective priming with emotional facial expression primes. Motiv. Emot. 35, 33-43. doi: 10.1007/s11031-010-9196-3

Baggio, H. C., Segura, B., Ibarretxe-Bilbao, N., Valldeoriola, F., Marti, M. J., Compta, Y., et al. (2012). Structural correlates of facial emotion recognition deficits in Parkinson's disease patients. Neuropsychologia 50, 2121-2128. doi: 10.1016/j.neuropsychologia.2012.05.020

Banse, R. (2001). Affective priming with liked and disliked persons: prime visibility determines congruency and incongruency effects. Cogn. Emot. 15, 501-520. doi: 10.1080/02699930126251

Baron-Cohen, S., Jolliffe, T., Mortimore, C., and Robertson, M. (1997). Another advanced test of theory of mind: evidence from very high functioning adults with autism or asperger syndrome. J. Child Psychol. Psychiatry 38, 813-822. doi: 10.1111/j.1469-7610.1997.tb01599.x 
Borg, C., Bedoin, N., Bogey, S., Michael, G. A., Poujois, A., Laurent, B., et al. (2012). Implicit and explicit emotional processing in Parkinson's disease. J. Clin. Exp. Neuropsychol. 34, 289-296. doi: 10.1080/13803395.2011.639296

Briesemeister, B. B., Kuchinke, L., and Jacobs, A. M. (2011a). Discrete emotion effects on lexical decision response times. PLoS ONE 6:e23743. doi: 10.1371/journal.pone.0023743

Briesemeister, B. B., Kuchinke, L., and Jacobs, A. M. (2011b). Discrete emotion norms for nouns: Berlin Affective Word List (DENN-BAWL). Behav. Res. Methods 43, 441-448. doi: 10.3758/s13428-011-0059-y

Buxton, S. L., MacDonald, L., and Tippett, L. J. (2013). Impaired recognition of prosody and subtle emotional facial expressions in Parkinson's disease. Behav. Neurosci. 127, 193-203. doi: 10.1037/a0032013

Calder, A. J., Young, A. W., Keane, J., and Dean, M. (2000). Configural information in facial expression perception. J. Exp. Psychol. Hum. Percept. Perform. 26, 527-551. doi: 10.1037/0096-1523.26.2.527

Calvo, M. G., and Marrero, H. (2009). Visual search of emotional faces: the role of affective content and featural distinctiveness. Cogn. Emot. 23, 782-806. doi $10.1080 / 02699930802151654$

Castner, J. E., Chenery, H. J., Copland, D. A., Coyne, T. J., Sinclair, F., and Silburn, P. A. (2007). Semantic and affective priming as a function of stimulation of the subthalamic nucleus in Parkinson's disease. Brain 130, 1395-1407. doi: 10.1093/brain/awm059

Challis, B. H., and Krane, R. V. (1988). Mood induction and the priming of semantic memory in a lexical decision task: asymmetric effects of elation and depression. Bull. Psychon. Soc. 26, 309-312. doi: 10.3758/BF03337666

Dagenbach, D., and Carr, T. H. (1994). "Inhibitory processes in perceptual recognition: evidence for a center-surround attentional mechanism," in Inhibitory Mechanisms in Attention, Memory and Language, eds D. Dagenbach and T. H. Carr (San Diego, CA: Academic Press), 412-443.

De Houwer, J., and Hermans, D. (1994). Differences in the affective processing of words and pictures. Cogn. Emot. 8, 1-20. doi: 10.1080/02699939408 408925

De Houwer, J., Hermans, D., and Eelen, P. (1998). Affective and identity priming with episodically associated stimuli. Cogn. Emot. 12, 145-169. doi: 10.1080/026999398379691

De Houwer, J., Teige-Mocigemba, S., Spruyt, A., and Moors, A. (2009). Implicit measures: a normative analysis and review. Psychol. Bull. 135, 347-368. doi: $10.1037 / \mathrm{a} 0014211$

Ekman, P., and Friesen, W. (1976). Pictures of facial Affect. Palo Alto, CA: Consulting Psychologists Press.

Estes, Z., and Verges, M. (2008). Freeze or flee? Negative stimuli elicit selective responding. Cognition 108, 557-565. doi: 10.1016/j.cognition.2008.03.003

Fazio, R. (2001). On the automatic activation of associated evaluations: an overview. Cogn. Emot. 15, 115-141. doi: 10.1080/02699930125908

Fox, E., Russo, R., and Dutton, K. (2002). Attentional bias for threat: evidence for delayed disengagement from emotional faces. Cogn. Emot. 16, 355-379. doi: 10.1080/02699930143000527

Giner-Sorolla, R., Garcia, M., and Bargh, J. A. (1999). The automatic evaluation of pictures. Soc. Cogn. 17, 76-96. doi: 10.1521/soco.1999.17.1.76

Glaser, J., and Banaji, M. R. (1999). When fair is foul and foul is fair: reverse priming in automatic evaluation. J. Pers. Soc. Psychol. 77, 669-687. doi: 10.1037/00223514.77.4.669

Hayes, C. J., Stevenson, R. J., and Coltheart, M. (2007). Disgust and Huntington's disease. Neuropsychologia 45, 1135-1151. doi: 10.1016/j.neuropsychologia. 2006.10.015

Hermans, D., Baeyens, F., and Eelen, P. (1998). Odours as affective-processing context for word evaluation: a case of cross-modal affective priming. Cogn. Emot 12, 601-613. doi: 10.1080/026999398379583

Hermans, D., De Houwer, J., and Eelen, P. (1994). The affective priming effect: automatic activation of evaluative information in memory. Cogn. Emot. 8, 515 533. doi: 10.1080/02699939408408957

Hermans, D., Spruyt, A., De Houwer, J., and Eelen, P. (2003). Affective priming with subliminally presented pictures. Can. J. Exp. Psychol. 57, 97-114. doi: 10.1037/h0087416

Hofmann, M. J., Kuchinke, L., Tamm, S., Võ, M. L.-H., and Jacobs, A. M. (2009). Affective processing within 1/10th of a second: high arousal is necessary for early facilitative processing of negative but not positive words. Cogn. Affect. Behav. Neurosci. 9, 389-397. doi: 10.3758/9.4.389
Ishai, A., Pessoa, L., Bikle, P. C., and Ungerleider, L. G. (2004). Repetition suppression of faces is modulated by emotion. Proc. Natl. Acad. Sci. U.S.A. 101, 9827-9832. doi: 10.1073/pnas.0403559101

Kissler, J., and Koessler, S. (2011). Emotionally positive stimuli facilitate lexical decisions - an ERP study. Biol. Psychol. 86, 254-264. doi: 10.1016/j.biopsycho.2010.12.006

Klapp, S. T. (2005). Two versions of the negative compatibility effect: comment on Lleras and Enns (2004). J. Exp. Psychol. Gen. 134, 431-435. doi: 10.1037/00963445.134.3.431

Klauer, K. C., and Musch, J. (2003). "Affective priming: findings and theories," in The Psychology of Evaluation, eds J. Musch and K. C. Klauer (Mahwah, NJ: Lawrence Erlbaum Associates), 7-51.

Krebs, J. F., Biswas, A., Pascalis, O., Kamp-Becker, I., Remschmidt, H., and Schwarzer, G. (2011). Face processing in children with autism spectrum disorder: independent or interactive processing of facial identity and facial expression? J. Autism Dev. Disord. 41, 796-804. doi: 10.1007/s10803-010-1098-4

Kuchinke, L., Jacobs, A. M., Grubich, C., Võ, M. L.-H., Conrad, M., and Herrmann, M. (2005). Incidental effects of emotional valence in single word processing: an fMRI study. Neuroimage 28, 1022-1032. doi: 10.1016/j.neuroimage.2005. 06.050

Kuchinke, L., Võ, M. L.-H., Hofmann, M., and Jacobs, A. M. (2007). Pupillary responses during lexical decisions vary with word frequency but not emotional valence. Int. J. Psychophysiol. 65, 132-140. doi: 10.1016/j.ijpsycho.2007. 04.004

Larsen, R. J., Mercer, K. A., Balota, D. A., and Strube, M. J. (2008). Not all negative words slow down lexical decision and naming speed: importance of word arousal. Emotion 8, 445-452. doi: 10.1037/1528-3542.8.4.445

Lee, S. Y., Kang, J. I., Lee, E., Namkoong, K., and An, S. K. (2011). Differential priming effect for subliminal fear and disgust facial expressions. Atten. Percept. Psychophys. 73, 473-481. doi: 10.3758/s13414-010-0032-3

Leppänen, J., and Hietanen, J. K. (2007). Is there more in a happy face than just a big smile? Vis. Cogn. 15, 468-490. doi: 10.1080/13506280600765333

Li, W., Zinbarg, R. E., Boehm, S. G., and Paller, K. A. (2008). Neural and behavioral evidence for affective priming from unconsciously perceived emotional facial expressions and the influence of trait anxiety. J. Cogn. Neurosci. 20, 95-107. doi: 10.1162/jocn.2008.20006

Maier, M. A., Berner, M. P., and Pekrun, R. (2003). Directionality of affective priming: effects of trait anxiety and activation level. Exp. Psychol. 50, 116-123. doi: $10.1026 / / 1618-3169.50 .2 .116$

Mattler, U. (2007). Inverse target-and cue-priming effects of masked stimuli. J. Exp. Psychol. Hum. Percept. Perform. 33, 83-102. doi: 10.1037/0096-1523. 33.1.83

Montagne, B., Kessels, R. P., Kammers, M. P., Kingma, E., de Haan, E. H., Roos, R. A., et al. (2006). Perception of emotional facial expression at different intensities in early-symptomatic Huntington's disease. Eur. Neurol. 55, 151-154. doi: $10.1159 / 000093215$

Murphy, S. T., and Zajonc, R. B. (1993). Affect, cognition and awareness: affective priming with optimal and suboptimal stimulus exposures. J. Pers. Soc. Psychol. 64, 723-739. doi: 10.1037/0022-3514.64.5.723

Nakic, M., Smith, B. W., Busis, S., Vythilingam, M., and Blair, R. J. R. (2006). The impact of affect and frequency on lexical decision: the role of the amygdale and inferior frontal cortex. Neuroimage 31, 1752-1761. doi: 10.1016/j.neuroimage.2006.02.022

Öhman, A., Lundqvist, D., and Esteves, F. (2001). The face in the crowd revisited: a threat advantage with schematic stimuli. J. Pers. Soc. Psychol. 80, 381-396. doi: 10.1037/0022-3514.80.3.381

Öhman, A., and Mineka, S. (2001). Fears, phobias and preparedness: toward an evolved module of fear and fear learning. Psychol. Rev. 108, 483-522. doi: 10.1037/0033-295X.108.3.483

Pinkham, A. E., Penn, D. L., Perkins, D. O., and Lieberman, J. (2003). Implications for the neural basis of social cognition for the study of schizophrenia. Am. J. Psychiatry 160, 815-824. doi: 10.1176/appi.ajp.160.5.815

Phillips, M. I., Young, A. W., Senior, C., Brammer, M., Andrew, C., Calder, A. J., et al. (1997). A specific neural substrate for perceiving facial expressions of disgust. Nature 389, 495-498. doi: 10.1038/39051

Rohr, M., Degner, J., and Wentura, D. (2012). Masked emotional priming beyond global valence activations. Cogn. Emot. 26, 224-244. doi: $10.1080 / 02699931.2011 .576852$ 
Sassi, F., Campoy, G., Castillo, A., Inuggi, A., and Fuentes, L. J. (2014). Task difficulty and response complexity modulate affective priming by emotional facial expressions. Q. J. Exp. Psychol. 67, 861-871. doi: 10.1080/17470218.2013.836233

Schacht, A., and Sommer, W. (2009). Time course and task dependence of emotion effects in word processing. Cogn. Affect. Behav. Neurosci. 9, 28-43. doi: 10.3758/CABN.9.1.28

Scott, G. G., O'Donnell, P. J., Leuthold, H., and Sereno, S. C. (2009). Early emotion word processing: evidence from event-related potentials. Biol. Psychol. 80, 95-104. doi: 10.1016/j.biopsycho.2008.03.010

Sprengelmeyer, R., Young, A. W., Calder, A. J., Karnat, A., Lange, H., Hömberg, V., et al. (1996). Loss of disgust. Perception of faces and emotions in Huntington's disease. Brain 119(Pt 5), 1647-1665. doi: 10.1093/brain/119.5.1647

Susa, G., Pitică, I., Benga, O., and Miclea, M. (2012). The self regulatory effect of attentional control in modulating the relationship between attentional biases toward threat and anxiety symptoms in children. Cogn. Emot. 26, 1069-1083. doi: 10.1080/02699931.2011.638910

Suslow, T., Dannlowski, U., Arolt, V., and Ohrmann, P. (2010). Adult attachment avoidance and automatic affective response to sad facial expressions. Aust. J. Psychol. 62, 181-187. doi: 10.1080/00049530903567203

Suslow, T., Ohrmann, P., Bauer, J., Rauch, A. V., Schwindt, W., Arolt, V., et al. (2006). Amygdala activation during masked presentation of emotional faces predicts conscious detection of threat-related faces. Brain Cogn. 61, 243-248. doi: 10.1016/j.bandc.2006.01.005

Verleger, R., Jaskowski, P., Aydemir, A., van der Lubbe, R. H. J., and Groen, M. (2004). Qualitative differences between conscious and nonconscious processing? On inverse priming induced by masked arrows. J. Exp. Psychol. Gen. 133, 494-515. doi: 10.1037/0096-3445.133.4.494

Võ, M. L.-H., Conrad, M., Kuchinke, L., Urton, K., Hofmann, M. J., and Jacobs, A. M. (2009). The Berlin Affective Word List Reloaded (BAWL-R). Behav. Res. Methods 41, 534-538. doi: 10.3758/BRM.41.2.534

Wang, K., Hoosain, R., Yang, R. M., Meng, Y., and Wang, C. Q. (2003). Impairment of recognition of disgust in Chinese with Huntington's or Wilson's disease. Neuropsychologia 41, 527-537. doi: 10.1016/S0028-3932(02)00171-9
Wang, S., Tudusciuc, O., Mamelak, A. N., Ross, I. B., Adolphs, R., and Rutishauser, U. (2014). Neurons in the human amygdala selective for perceived emotion. Proc. Natl. Acad. Sci. U.S.A. 111, 3110-3119. doi: 10.1073/pnas.1323342111

Wentura, D., Rothermund, K., and Bak, P. (2000). Automatic vigilance: the attention-grabbing power of approach- and avoidance-related social information. J. Pers. Soc. Psychol. 78, 1024-1037. doi: 10.1037/0022-3514.78.6.1024

Williamson, S., Harpur, T. J., and Hare, R. D. (1991). Abnormal processing of affective words by psychopaths. Psychophysiology 28, 260-273. doi: 10.1111/j.1469-8986.1991.tb02192.x

Windmann, S., Daum, I., and Güntürkün, O. (2002). Dissociating prelexical and postlexical processing of affective information in the two hemispheres: effects of the stimulus presentation format. Brain Lang. 80, 269-286. doi: 10.1006/brln.2001.2586

Yang, E., Zald, D. H., and Blake, R. (2007). Fearful expressions gain preferential access to awareness during continuous flash suppression. Emotion 4, 882-886. doi: 10.1037/1528-3542.7.4.882

Conflict of Interest Statement: The authors declare that the research was conducted in the absence of any commercial or financial relationships that could be construed as a potential conflict of interest.

Received: 03 July 2014; accepted: 30 August 2014; published online: 17 September 2014 Citation: Wagenbreth C, Rieger J, Heinze H-J and Zaehle T (2014) Seeing emotions in the eyes - inverse priming effects induced by eyes expressing mental states. Front. Psychol. 5:1039. doi: 10.3389/fpsyg.2014.01039

This article was submitted to Emotion Science, a section of the journal Frontiers in Psychology.

Copyright () 2014 Wagenbreth, Rieger, Heinze and Zaehle. This is an open-access article distributed under the terms of the Creative Commons Attribution License (CC BY). The use, distribution or reproduction in other forums is permitted, provided the original author(s) or licensor are credited and that the original publication in this journal is cited, in accordance with accepted academic practice. No use, distribution or reproduction is permitted which does not comply with these terms. 